\title{
EFECTO DE LACTOBACILLUS DELBRUECKII SOBRE LAS PROPIEDADES TEXTURALES DE GELES BINARIOS DE GOMA GELANA
}

\section{EFFECT OF LACTOBACILLUS DELBRUECKII ON THE TEXTURAL PROPERTIES OF BINARY GELS OF GELLAN GUM}

\author{
*González C. Rafael E., Pérez M. Jaime., Tarón D. Arnulfo. \\ Universidad de Cartagena, Facultad de Ingeniería. Piedra de Bolívar - Av Del Consulado, Calle $30 N^{\circ} 48$ - \\ 157., Cartagena D.T y C. Colombia. Correo electrónico: *rgonzalezc1@unicartagena.edu.co
}

Recibido 25 de Julio 2015; aceptado 30 de octubre de 2015

\section{RESUMEN}

El objetivo del presente trabajo fue evaluar el efecto de la adición de una bacteria probiótica sobre las propiedades texturales de geles comestibles elaborados a base de goma gelana. Para la elaboración de la biopelículas se utilizó un diseño de mezclas simple así: gelana de alto acilo (GAA), gelana de bajo acilo (GBA) y sus mezclas 25GAA/75GBA, 50GAA/50GBA, 75GAA/25GBA, con adición de Lactobacillus delbrueckii. Posteriormente se determinaron las propiedades texturales. Los resultados mostraron que ninguna de las propiedades texturales (dureza, adhesividad, cohesividad, elasticidad) resultan afectadas por la adición de la bacteria probiótica. Sin embargo, todas las formulaciones presentaron características texturales apropiadas para el crecimiento de la bacteria probiótica durante su almacenamiento. Por consiguiente, los geles con adición de bacterias probióticas pueden ser aplicados a una gran variedad de productos alimenticios para incrementar su opción de consumo. 
*Autor a quien debe dirigirse la correspondencia. Correo Electronico: rgonzalezc1@unicartagena.edu.co
Palabras Claves: Gelana de alto acilo, Gelana de bajo acilo, geles comestibles, Lactobacillus delbrueckii.

\section{ABSTRACT}

The aim of this study was to evaluate the effect of adding a probiotic bacterium on the textural properties of edible gels prepared from gellan gum. Simple design mixtures was used for the development of the gels: high acyl gellan (HAG), low acyl gellan (LAG) and their mixtures 25HAG / 75LAG, 50HAG / 50LAG, 75HAG / 25LAG, with addition of Lactobacillus delbrueckii. Results showed that none of the textural properties (hardness, adhesiveness, cohesiveness, elasticity) were affected by the probiotic addition. However, all formulations showed appropriate textural characteristics for the growth of probiotic bacteria during storage. Therefore, gels with added probiotic bacteria could be applied to a variety of foods and to increase their consumer choice.

Keywords: High acyl gellan, Low acyl gellan, glycerol, edible gels, Lactobacillus delbrueckii.

\section{INTRODUCCIÓN}

\section{Las bacterias probióticas son} microorganismos vivos que proporcionan efectos favorables a la salud de los consumidores. Algunos de estos efectos incluyen: control del colesterol, regulación de infecciones intestinales, mejora del sistema inmunológico, utilización de lactosa y disminución de actividad anticancerígena (Zhu y col, 2011; Tripathi y Giri, 2014; Cornelius y col, 2002; De las Caigigas 2002;
Sungsoo y Finocchiaro, 2010). Por consiguiente, productos que suministren este tipo de bacterias deberían ser parte de una dieta alimentaria saludable. La federación internacional láctea (IDF por sus siglas en inglés) recomienda una dosis mínima de $10^{6} \mathrm{UFC} / \mathrm{g}$ para que los efectos de las bacterias sean percibidos por los consumidores. 
En muchos casos el consumo de bacterias probióticas se ve limitado debido al limitado acceso que tienen los productos lácteos (Röble y col, 2010; Olaiz-Fernández, 2012). Por tal motivo, la incorporación de probióticos en la dieta ha despertado el interés en la búsqueda de nuevas aplicaciones alimentarias, conllevando desarrollo de alimentos funcionales (Röble y col, 2010; Sungsoo y Finocchiaro, 2010; Olaiz-Fernández, 2012). Dentro de las investigaciones llevadas a cabo para incrementar la concentración de bacterias probióticas en sistemas alimentarias se han estudiado su aplicación en películas comestibles (García-Argueta, 2013) y procesos de microencapsulación (González y col, 2014; González y col, 2015) utilizando polímeros funcionales como la goma gelana.

La goma gelana, es un Heteropolisacárido lineal aniónico producido por la bacteria Sphingomonas paucimobilis y consiste de unidades de repetición de un tetrasacárido (1,3- $\beta$-D-Glucosa;

1,4- $\beta$-D-Acido glucurónico; 1,4 $\beta-D$ Glucosa; y 1,4 a-Lramnosa), (González y col, 2012). La gelana nativa es conocida como gelana de alto acilo (GAA) debido a que presenta un grupo acetato y glicerato en su residuo de glucosa-A (González, 2011).
Cuando la GAA es expuesta a un fuerte tratamiento con álcali a elevadas temperaturas, los grupos acilo son hidrolizados, obteniendo de esta manera la goma gelana de bajo acilo (GBA), (Mao y col, 2000) que se caracteriza por formar geles más estables en comparación a la GAA (González y col, 2011). Pese a ello, no se ha estudiado el efecto de bacterias probióticas sobre geles poliméricos a base de goma gelana.

Aunque el uso de bacterias probióticas como componentes en sistemas poliméricos continúa (Tapia y col, 2007; Rojas-Graü y col, 2009; Ramírez y col, 2012).

La adición de bacterias probióticas en geles comestibles puede ser una alternativa en el consumo de probióticos. Por lo mencionado anteriormente el objetivo del presente trabajo fue analizar el efecto de la adición de una bacteria probiotica (Lactobacillus delbrueckii) en las propiedades texturales de un gel polimérico binario a base de gelana de alto y bajo acilo. 


\section{MATERIALES Y MÉTODOS}

\section{Materiales}

La $\delta$-gluconolactona, la goma gelana de altoy bajo acilo fue suministrada por modernist pantry (USA). El glicerol ( $87 \%$ de pureza) y el Carbonato de Calcio por Merck (USA).

\section{Preparación de los geles}

Las dispersiones de goma gelana al $0.2 \%$ $(p / v)$ que es recomendada para utilizar en productos alimenticios (Kelco, 1995) GAA, GBA y sus mezclas 25GAA/75GBA, 50GAA/50GBA, 75GAA/25GBA fueron disueltas bajo agitación constante a $90^{\circ} \mathrm{C}$ en presencia de calcio $(30 \mathrm{mM})$ y glicerol $5 \%(\mathrm{v} / \mathrm{v})$. Posteriormente las dispersiones fueron acidificadas con $\delta$-gluconolactona hasta un $\mathrm{pH}$ de 4.5 para liberar el ion calcio por reacción de sustitución de la sal de calcio utilizada $\left(\mathrm{CaCO}_{3}\right)$

\section{Propiedades texturales}

Un texturometro Shimadzu modelo EZ-Test EZ-S A fue utilizado para la determinación de las propiedades texturales. Las muestras de los geles fueron de geometría cilíndrica de $3 \mathrm{~cm}$ de diámetro y $4 \mathrm{~cm}$ de espesor. Las muestras fueron colocadas entre dos placas metálicas. La velocidad de la placa superior para el análisis fue de $30 \mathrm{~mm} / \mathrm{s}$. cada análisis se realizó 5 veces.

\section{Tratamiento de los datos}

Las propiedades texturales (dureza, elasticidad, cohesividad y adhesividad) fueron sometidos a un análisis normal de varianza (ANOVA-un factor). $\mathrm{La}$ comparación entre los promedios aritméticos se llevó a cabo mediante la prueba de tukey. El análisis fue realizado por medio del programa de computo SPSS ver 17 para Windows.

\section{RESULTADOS Y DISCUSIÓN}

\section{Elaboración de geles}

Se obtuvieron geles de $3 \mathrm{~cm}$ de diámetro y $4 \mathrm{~cm}$ de espesor. Sin embargo, los geles con una concentración mayor al $50 \%(\mathrm{p} / \mathrm{v})$ de gelana de alto acilo no podían mantener su forma una vez eran retirados los moldes, similares resultados fueron obtenidos por González y col, (2013) en la obtención de microcápsulas utilizando mezclas binarias de gelana de alto y bajo acilo, ya que la gelana de alto acilo origina geles bastante elásticos como consecuencia del impedimento estérico que ejercen los 
grupos acilo frenando la reacción de los grupos funcionales con el ión calcio (González y col, 2012).

\section{Análisis de textura de los geles}

Se encontró que la GAA y la GBA tienen un efecto notorio $(P<0.05)$ en la dureza, cohesividad, elasticidad y adhesividad de los geles, mientras que la adición de Lactobcillus delbrueckii no tiene ningún efecto sobre las propiedades texturales, ya que no se presentaron diferencias $(P<0.05)$ con respecto a los geles que no contenían la bacteria probiótica, estos resultados son contrarios a los reportados por GarcíaArgueta y col, (2013) quienes observaron diferencias en atributos texturales como la dureza al adicionar una bacteria probiótica a biopelículas elaboradas a base de proteína, glicerol e inulina; esta diferencia probablemente es ocasionada por el crecimiento de la bacteria probiótica al utilizar la proteína como sustrato. Por el contrario, los resultados que aquí se presentan demuestran que la bacteria utilizada es incapaz de utilizar las gomas gelanas como fuente de carbono debido a que son polímeros complejos y se encuentran formando una red tridimensional (González y col, 2012) lo cual dificulta su degradación y posterior aprovechamiento celular.
Tabla 1. Valores promedio de las propiedades texturales de los geles de gelana

\begin{tabular}{|c|c|c|c|c|c|}
\hline GBA & GAA & $\begin{array}{l}\text { Dureza } \\
\text { (N) }\end{array}$ & $\begin{array}{c}\text { Elasticid } \\
\text { ad }\end{array}$ & $\begin{array}{c}\text { Cohesivi } \\
\text { dad }\end{array}$ & $\begin{array}{c}\text { Adhesivi } \\
\text { dad(g/m } \\
m)\end{array}$ \\
\hline 0 & 100 & $2.24 \pm 0.4^{\mathrm{a}}$ & $0.95 \pm 1.2^{\mathrm{a}}$ & $0.54 \pm 3.4^{a}$ & $\begin{array}{c}- \\
2.34 \pm 1.6^{\mathrm{a}}\end{array}$ \\
\hline 25 & 75 & $3.45 \pm 2.1^{b}$ & $0.76 \pm 2.3^{b}$ & $0.62 \pm 2.8 b$ & $\begin{array}{c}- \\
2.18 \pm 1.1^{\mathrm{b}}\end{array}$ \\
\hline 50 & 50 & $3.65 \pm 1.8^{b}$ & $0.45 \pm 2.3^{c}$ & $0.64 \pm 1.8 \mathrm{~b}$ & $\begin{array}{c}- \\
1.67 \pm 1.7^{c}\end{array}$ \\
\hline 75 & 25 & $4.55 \pm 1.8^{c}$ & $0.27 \pm 2.7^{\mathrm{d}}$ & $0.72 \pm 2.4 \mathrm{c}$ & $\begin{array}{c}- \\
1.58 \pm 0.5^{\mathrm{d}}\end{array}$ \\
\hline 100 & 0 & $6.78 \pm 2.8^{d}$ & $0.23 \pm 1.6^{\mathrm{d}}$ & $0.75 \pm 2.4 \mathrm{c}$ & $\begin{array}{c}- \\
1.23 \pm 1.4^{\mathrm{e}}\end{array}$ \\
\hline \multicolumn{6}{|c|}{ Con adición de Lactobacillus delbrueckii } \\
\hline 0 & 100 & $2.28 \pm 0.2^{\mathrm{a}}$ & $0.87 \pm 2.4^{\mathrm{a}}$ & $0.49 \pm 2.2^{\mathrm{a}}$ & $\begin{array}{c}- \\
3.12 \pm 0.4^{\dagger}\end{array}$ \\
\hline 25 & 75 & $3.41 \pm 3.1^{b}$ & $0.78 \pm 1.3^{b}$ & $0.66 \pm 1.3^{b}$ & $2.05 \pm 3.1^{\mathrm{b}}$ \\
\hline 50 & 50 & $3.75 \pm 1.2^{b}$ & $0.55 \pm 3.0^{c}$ & $0.73 \pm 1.4^{c}$ & $\begin{array}{c}- \\
1.76 \pm 1.3^{\mathrm{g}}\end{array}$ \\
\hline 75 & 25 & $4.45 \pm 1.7^{c}$ & $0.35 \pm 1.1^{\mathrm{e}}$ & $0.70 \pm 3.1^{c}$ & $\begin{array}{c}- \\
1.54 \pm 1.8^{d}\end{array}$ \\
\hline 100 & 0 & $6.58 \pm 1.4^{\mathrm{d}}$ & $0.18 \pm 1.3^{\dagger}$ & $0.77 \pm 2.8^{c}$ & $\begin{array}{c}- \\
1.22 \pm 1.2^{\mathrm{e}}\end{array}$ \\
\hline
\end{tabular}

Valores de las filas sin ninguna letra en común presentaron diferencias significativas a un nivel de confianza del $95 \%$

La elasticidad de los geles incrementa significativamente $(P<0.05)$ con el aumento en la proporción de GAA, debido a que en la GAA se establecen enlaces hidrógeno intermoleculares principalmente. La dureza por el contrario incrementa con en aumento en la proporción de GBA como consecuencia de la formación de enlaces iónicos intermoleculares presentes. En términos generales, la GAA no requiere 
calcio para iniciar el proceso de gelificación solamente necesita de un aumento y disminución de la temperatura para gelificar. Caso contrario a la GBA que si requiere de cationes para iniciar su proceso de gelificación debido a la unión de cadenas lineales por la presencia de calcio.

Con respecto a la cohesividad, se pudo apreciar una disminución $(P<0.05)$ de esta propiedad con el incremento de GAA. En contraste, la adhesividad fue directamente proporcional a la concentración de GAA utilizada.

Aunque se ha reportado que la adición de bacterias ácido lácticas puede debilitar la estructura tridimensional de películas comestibles por las interacciones entre cadenas poliméricas conllevando a una disminución de la flexibilidad y un incremento de la fragilidad de las películas.

Villagomez-Zavala y col, (2008) mencionaron que dichas características pueden estar relacionadas al tipo de estructuras involucradas en la gelación de los multicomponentes presentes en los geles o películas.

\section{Viabilidad de Lactobacillus delbrueckii}

La viabilidad de Lactobacillus delbrueckii fue realizada a través de microbiología tradicional utilizando agar MRS para su enumeración. Los resultados obtenidos de pueden apreciar en la tabla 2, donde se puede observar que la concentración de bacterias se mantiene constante durante quince días de almacenamiento, lo cual indica que las propiedades texturales y las relaciones de gelana utilizadas no tienen efecto sobre la viabilidad bacteriana.

Tabla 2. Concentración de Lactobacillus delbrueckii (UFC/g) en los geles

\begin{tabular}{|c|c|c|c|}
\hline GAA & GBA & $\begin{array}{c}\text { Día 1 } \\
\text { (Log10UFC/g) }\end{array}$ & $\begin{array}{c}\text { Día 15 } \\
\text { (Log10UFC/g) }\end{array}$ \\
\hline 0 & 100 & 8.54 & 8.33 \\
\hline 25 & 75 & 8.47 & 8.52 \\
\hline 50 & 50 & 8.37 & 8.75 \\
\hline 75 & 25 & 8.52 & 8.60 \\
\hline 100 & 0 & 8.51 & 8.81 \\
\hline
\end{tabular}

Es importante mencionar que el mantenimiento de la viabilidad bacteriana puede ser ocasionado también por la presencia de glicerol, que es normalmente utilizado como conservante de bacterias benéficas. Por tal motivo, los geles de gelana conteniendo glicerol pueden proporcionar una alternativa en el suministro de bacterias benéficas en una dieta alimentaria saludable 


\section{CONCLUSIONES}

Es posible extraer pectina a partir de la cáscara de limón Tahití, por lo tanto es necesario conocer las condiciones que puedan aumentar el rendimiento del proceso y tener efecto directo sobre la calidad de la pectina.

No existen coincidencias en las condiciones de extracción para los dos estados, así que se deben manejar tratamientos diferentes en lo respectivo a condiciones de tiempo y temperatura; para el factor $\mathrm{pH}$ se debe considerar el manejo de valores en un rango bajo (1.5 - 3.5), ya que a estos rangos se obtiene pectina de alto metoxilo y por encima de estos valores no hay gelificación.

Se lograron establecer las características de la pectina para ambos estados de maduración y se demostró que existe diferencia entre la pectina obtenida a partir de la cáscara de limón verde y la pectina patrón en todas las características de calidad.
Existe menos diferencia entre la pectina obtenida a partir de cáscara de limón Tahití maduro en comparación a la pectina Patrón.

El alto porcentaje de ácido galacturónico y el bajo porcentaje de cenizas en la pectina, son dos de los criterios que evalúan la pureza de las pectinas, porque se evidencia que las pectinas extraídas, son de alta pureza y aptas para ser usadas en la Industria.

\section{REFERENCIAS BIBLIOGRÁFICAS}

Abbott SL, Cheung KW. Janda M. (2003).

The genus Aeromonas: Biochemical characteristics, atypical reactions, and phenotypic identification schemes. J Clin Microbiol; 37(11):2348-2357.
Balaji V, Jesudason V, Sridharan G. (2004). Cytotoxin testing of environmental Aeromonas spp. In Vero cell culture. Indian J Med Res; 119:186-189.

Cabrera LE, Bravo L, Ramírez MM, Llop A, Fernández A, Morier L, Borrego G. 
@LIMENTECH CIENCIA Y TECNOLOGÍA ALIMENTARIA ISSN 1692-7125. Volumen 13, No. 2, p. 216-225, año 2015 Facultad de Ingenierías y Arquitectura Universidad de Pamplona

(2007). Factores de virulencia en cepas de Aeromonas spp. Aisladas de pacientes con bacteriemia. Rev PanamInfectol 2007; 9(4):19-23.

Cascón A, Yugueros J, Temprano A, Sánchez M, Herranz C, Luengo JM. (2000). A major secreted enastase is essential for pathogenicity of Aeromonas hydrophila. Infect Immun; 68:3233-3241.

Castilho, M.C.; Castro, T.L.; Araújo, V.S.; Trajano, R.S.; Santos, P.A.; Pimenta, P.M.; Lucheze, K.; Melo, J.T.; Gonçalves, A.M.; Nogueira, R.T.; de Luna, M.G.; Freitas-Almeida, A.C. (2009). High frequency of hemolytic and cytotoxic activity in Aeromonas spp. isolated from clinical, food and environmental in Rio de Janeiro, Brazil. Antonie Van Leeuwenhoek; 96:53-61.

Castro-Escarpulli G, Aguilera-Arreola M, Giono S, Hernández-Rodríguez C, Rodríguez M, Soler L, Aparicio, G.; Figueras, M. (2002). El género Aeromonas. ¿Un patógeno importante en México? Enf Infec Micro; 22(4):206-216.

Castro-Escarpulli G, Peña del Barrio D, Castañeda N, García A, Morier L, Aguilera-Arreola MG, Bravo L. (2002). Virulence factors of $A$. caviae strains isolated from acute diarrheic disease in
Cuba. Rev Latinoam Microbiol; 44(1):1113.

Chacón MR, Figueras MJ, Castro-Escarpulli G, Soler L, Guarro J. (2003). Distribution of virulence genes in clinical and environmental isolates of Aeromonas spp. Antonie Van Leeuwenhoek; 84:269278.

Farmer JJ, Arduino MJ, Hickman-Brenner FW. (2006). The genera Aeromonas and Plesiomonas. Prokaryotes; 6:564-596.

González-Serrano CJ, Santos JA, García López ML y Otero A. (2002) Virulence markers in Aeromonas hydrophila and Aeromonas veronii biovar sobria isolates from freshwater fish and from a diarrhoea case. J Appl Microbiol; 93:414-419.

Guerra MF, Fadanelli R, Figueiró $M$, Schreiner F, Delamare AP, Wollheim C, Costa SP, Echeverrigaray S. (2007). Aeromonas associated diarrhoeal disease in south Brazil: prevalence, virulence factors and antimicrobial resistance. Braz J Microbiol; 38(4):638643.

INVIMA. (2008) Ministerio de la Protección social. Informe de la vigilancia de las enfermedades transmitidas por alimentos. [en línea]. [Consultado Mayo 16 de 2011] URL Disponible En: 
(a)LIMENTECH CIENCIA Y TECNOLOGÍA ALIMENTARIA ISSN 1692-7125. Volumen 13, No. 2, p. 216-225, año 2015 Facultad de Ingenierías y Arquitectura Universidad de Pamplona

\section{http://web.}

invima.gov.co/portal/documents/portal/do cuments/root/INFORMEETA_\%20

IITRIMESTRE\%20200801.pdf.

Ivanova EP, Zhukova NV, Gorshkova NM, Chaikina EL. (2001) Characterization of Aeromonas and Vibrio species isolated from a drinking water reservoir. J Appl Microbiol; 90:919-927.

Janda JM, Abbott SL. (2010) The genus Aeromonas:Taxonomy, pathogenicity, and infection. Clin Microbiol Rev; 23:3573.

Krzyminska S, Mokracka J, Laganowska M, Wlodarczak K, Guszczynska E, Liszkowska J, Popkowska E, Lima I, Lemanska I, Wendt M. (2001) Enhancement of the virulence of Aeromonas caviae diarrhoeal strains by serial passages in mice. J Med Microbiol; 50:303-312.

Longa A, Vizcaya L, Nieves B, Bravo L, Morier L, Pérez-Schael I, Cabrera LE. (2005) Factores de virulencia asociados a la enteropatogenicidad en cepas de Aeromonas spp. aisladas de niños con diarrea en Mérida, Venezuela. Rev Cubana Med Trop; 57(2):85-91.

Martin-Carnahan A. Joseph SW. (2005) Aeromonadaceae. En: Brenner DJ, Krieg
NR, Staley JT, Garrity GM. Eds. The Proteobacteria, Part B, Bergey's Manual of Systematic Bacteriology. 2nd Ed. NewYork, NY: Springer-Verlag.

Merino S, Aguilar A, Nogueras MM, Regue M, Swift S, Tomas JM. (1999). Cloning, sequencing, and role in virulence of two phospholipases (A1 and C) from mesophilic Aeromonas sp. serogroup O:34. Infect Immun; 67(8):4008-4013.

Rahman M, Colque-Navarro P, Kunh I, Huys G, Swings J, Möllby R. (2002). Identification and characterization of pathogenic Aeromonas veronii Biovar sobria associated with epizootic ulcerative syndrome in fish in Bangladesh. Appl Environ Microbiol; 68(2):650-655.Resistance. Emerg Infect Dis; 9(5):552-555.

Santos A, González J, Otero A, GarcíaLópez ML. (1999). Hemolytic activity and siderophore production in different Aeromonas species isolated from fish. Appl Environ Microbiol; 65(12):56125614.

Sechi LA, Deriu A, Falchi MP, Fadda G y Zanetti S. (2002). Distribution of virulence genes in Aeromonas spp. isolated from Sardinian waters and from patients with diarrhea. J App Microbiol; 92:221-227. 
Soler L, Figueras MJ, Chacón MR, Vila J, Marco F, Martinez-Murcia AJ, Guarro J. (2002). Potential virulence and antimicrobial susceptibility of Aeromonas popoffii recovered from freshwater and seawater. FEMS Immunol Med Microbiol; 32:243-247.

Vila J, Ruiz J, Gallardo F, Vargas M, Soler L, Figueras MJ, Gascón J. (2003) Aeromonas spp. and traveler's diarrhea: Clinical Features and Antimicrobial

Von Graevenitz A. (2007). The role of Aeromonas in diarrhea: a review. Infection; 35(2):59-64.

Yucel N, Erdogan S (2010). Virulence properties and characterization of aeromonads isolated from foods of animal origin and environmental sources. J Food Prot; 73(5):855-60. 